Presented at: Accelerator Based Sources of

CONF-970706-Infrared and Applications

San Diego, CA

July 29-30, 1997

BNL-64880

\title{
Industrial Applications of Accelerator-Based Infrared Sources Analysis Using Infrared Microspectroscopy
}

\author{
Jean-Louis Bantignies, Gilbert Fuchsa, G. L. Carr \\ Paul Dumas, and Catherine Wilhelm ${ }^{2}$
}

'Elf Atochem, CRRA, Rue Henry Moissan, B.P. 63-69 493 Pierre-Benite Cedex, France

'National Synchrotron Light Source, Brookhaven National Laboratory, Upton, NY 11973-5000

'LURE, Bat 209 D, Centre Universitaire Paris-Sud, 91405 ORSAY Cedex, France

\section{RECEIVED \\ DEC 031997 \\ OSTI}

September 1997

DISTRIBUTION OF THIS DOCUMENT IS UNLRPITED

National Synchrotron Light Source

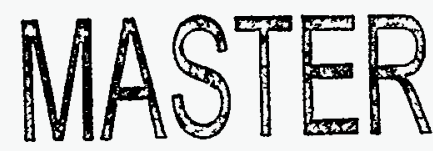

Brookhaven National Laboratory

Upton, NY 11973

Work performed under the auspices of the U.S. Department of Energy, under contract DE-AC02-76CH00016 


\section{DISCLAIMER}

This report was prepared as an account of work sponsored by an agency of the United States Government. Neither the United States Government nor any agency thereof, nor any of their employees, makes any warranty, express or implied, or assumes any legal liability or responsibility for the accuracy, completeness, or usefulness of any information, apparatus, product, or process disclosed, or represents that its use would not infringe privately owned rights. Reference herein to any specific commercial product, process, or service by trade name, trademark, manufacturer, or otherwise does not necessarily constitute or imply its endorsement, recommendation, or favoring by the United States Government or any agency thereof. The views and opinions of authors expressed herein do not necessarily state or reflect those of the United States Government or any agency thereof. 


\section{DISCLAMIER}

Portions of this doement may be illegible in electronic image produets. Innges are produced from the best available original docementent. 


\title{
Industrial applications of accelerator-based infrared sources Analysis using infrared microspectroscopy
}

\author{
Jean-Louis Bantignies ${ }^{\mathrm{a}}$, Gilbert Fuchs ${ }^{\mathrm{a}}$, G. L. Carr ${ }^{\mathrm{b}}$, Paul Dumas ${ }^{\mathrm{c}}$, Catherine Wilhelm ${ }^{\mathrm{a}}$ \\ ${ }^{a}$ Elf Atochem, CRRA, Rue Henry Moissan -B. P. 63- 69493 Pierre-Bénite Cedex France \\ bNational Synchrotron Light Source, Brookhaven National Laboratory, Upton, New York 11973 \\ ${ }^{c}$ LURE, Bat 209 D, Centre Universitaire Paris-Sud, 91405 ORSAY cedex, France
}

\begin{abstract}
Infrared Microspectroscopy, using a globar source, is now widely employed in the industrial environment, for the analysis of various materials. Since synchrotron radiation is a much brighter source, an enhancement of an order of magnitude in lateral resolution can be achieved. Thus, the combination of IR microspectroscopy and synchrotron radiation provides a powerful tool enabling sample regions only few microns size to be studied. This opens up the potential for analyzing small particles. Some examples for hair, bitumen and polymer are presented.
\end{abstract}

Keywords: infrared microspectroscopy, synchrotron, hair, bitumen, polymer

\section{INTRODUCTION}

Mid-IR spectroscopy (between 4000 and $650 \mathrm{~cm}^{-1}$ ) is widely employed for the identification of molecular systems because of its high chemical specificity. To characterize samples at the microscopic level, however, a molecular microprobe device is desirable. At the end of the 80 's infrared spectrometers were coupled with optical microscopes to allow infrared microspectroscopy measurements to be made ${ }^{i}$. Such systems have now been developed to the point where they are now widely used for industrial applications ${ }^{2}$.

Whereas conventional visible light microscopy is useful for identifying physical microstructure, IR microspectroscopy provides chemical imaging of the sample.

Infrared imaging is achieved by raster scanning the sample across the microfocused IR beam, taking a complete spectrum at each point. From these data a map of the integrated absorbance of a specific frequency is built up. In an IR microscope, the lateral resolution of such an image is imposed by the projected size of the aperture, which itself is limited by the brightness of the IR source. Indeed, for mapping experiments, a conventional infrared source (typically a $1200 \mathrm{~K}$ blackbody) does not possess sufficient brightness to achieve acceptable signal-to-noise $(\mathrm{S} / \mathrm{N})$ ratios and reasonable scanning times when the size of the beam on the sample is less thian $30 \times 30$ microns. In order to improve the lateral resolution, herefore, a brighter source is needed. Infrared light produced as synchrotron radiation has been found to be 2 to 3 orders of magnitude brighter than conventional sources ${ }^{3}$. Since 1994 at the NSLS, Brookhaven, an infrared microspectrometer has been coupled to a synchrotron infrared light source ${ }^{4}$. This has allowed mapping experiments with an acceptable S/N (-Root Mean SquareRMS $(\mathrm{T} \%)=5 \times 10^{-3}$ ) with a beam size of only $6 \times 6$ microns. Further improvements are possible, depending on the absorbance and thickness of the material.

The present work was done at the National Synchrotron Light Source (Brookhaven National Laboratory) at the IR micro spectroscopy beamline in the frame of collaboration with the Elf Aquitaine Company.

In this paper, we focuse first on the comparison between conventional and synchrotron sources. Then, we also report results of chemical imaging characterization obtained in different fields of interest of the chemical industry. In cosmetics, results obtained on the microstructural characterization of hair are presented. Bitumen and polymers infrared imaging characterizations are also reported.

Further author information -

G.S.C.(correspondence): Email: Bantigni@bnllsl.nsls.bnl.gov; Fax :516 3443238 


\section{SYNCHROTRON INFRARED SPECTROSCOPY}

A commercial IR microspectrometer (a Spectra -Tech "Ir $\mu \mathrm{s}$ "tm) is installed at beamline U2B ${ }^{3,4}$ of the NSLS. The Irus system consists of an integated FTR spectrometer and microscope optical module. The spectrometer contains a rapid-scan Michelson interferometer with a germanium coated $\mathrm{KBr}$ beam splitter. Spectral resolution can selected from 2 to $16 \mathrm{~cm}^{-1}$. It is equipped with a MCT ( $\mathrm{HgCdTe}$ ) detector which allows experiments between 4000 to $600 \mathrm{~cm}^{-1}$. The microscope optics used was a $32 x, 0.65 \mathrm{NA}$, objective lens. The condenser used for the transmission experiments was a 10X, $0.71 \mathrm{NA}$. Both objective and condenser were Schwarzschild reflecting designs. A two dimensional motorized stage was used for the raster scanning. A schematic of the Ir mis microspectrometer is shown in figure 1.

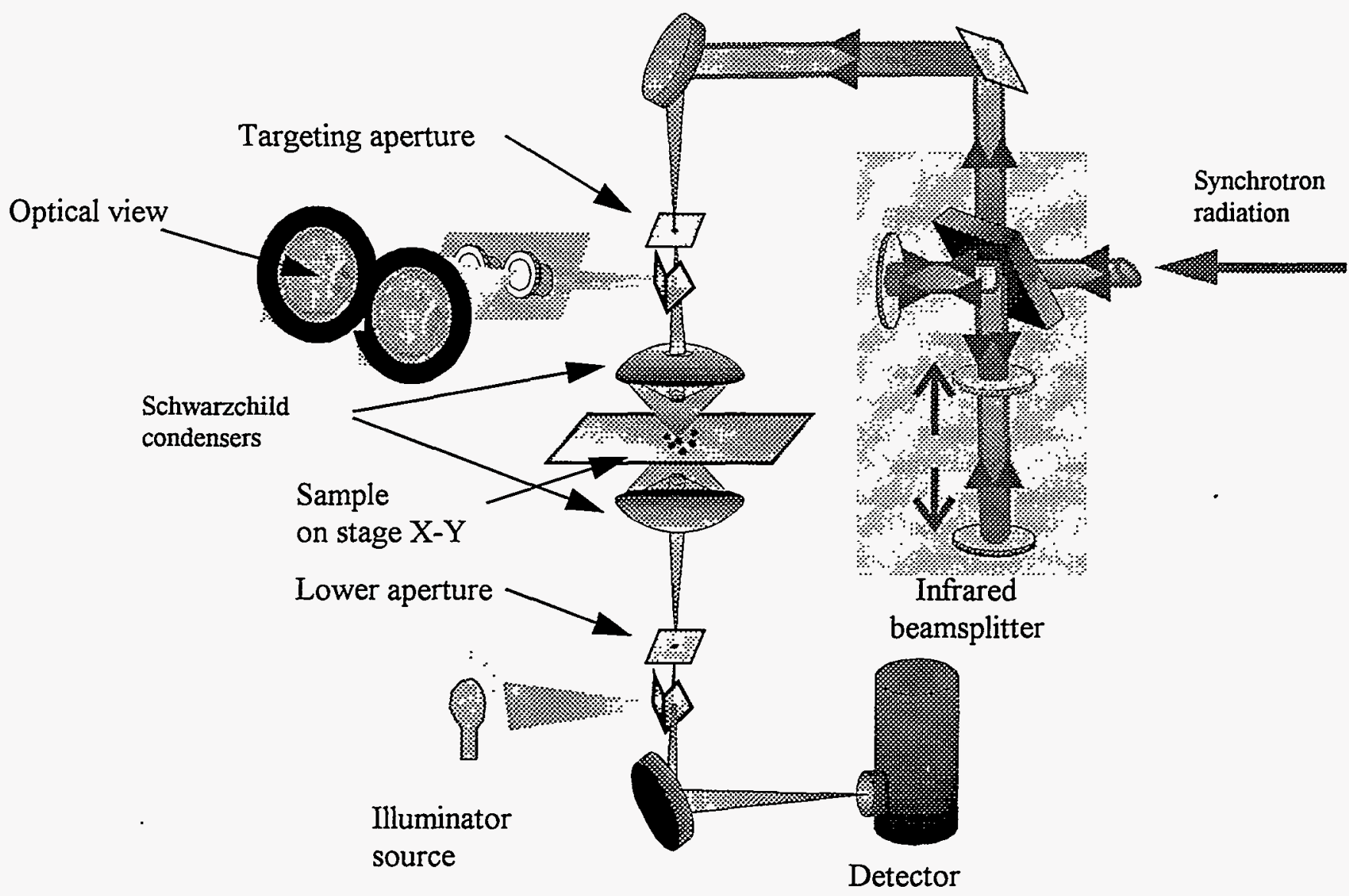

Figure 1: Schematic diagram of the Ir $\mu$ s scanning infrared microspectrometer.

The higher brightness of the synchrotron source compared to the globar in the mid-IR region has been quantified at the NSLS for the U2B beamline $e^{3}$, the U2/globar ratio being about $10^{2}$ at $1000 \mathrm{~cm}^{-1}$. This enhancement of the brightness allows regions smaller than $10 \mu \mathrm{m}$ to be probed with acceptable $\mathrm{S} / \mathrm{N}$ values. We quantified the $\mathrm{S} / \mathrm{N}$ of the microspectrometer with the synchrotron source and the globar source by ratioing two nominally identical spectra $\left(128 \mathrm{scans} / 4 \mathrm{~cm}^{-1}\right)$ through a $2 \mathrm{~mm}$ thick $\mathrm{BaF}_{2}$ pellet. The plots of the ratios (versus frequency) produces a line around $100 \%$, the deviations from $100 \%$ giving the lack of reproducibility, or noise. The RMS value of the "noise" was evaluated in $500 \mathrm{~cm}^{-1}$ intervals from 4000 $\mathrm{cm}^{-1}$ to the lowest frequency usable. With a beam size of $6 \times 6$ microns for both sources, the synchrotron/globar noise ratio is about $10^{-2}$ between 3000 and $2500 \mathrm{~cm}^{-1}$, which is in agreement with the brightness calculations. Addressing practical 
limitations of the spatial resolution, however, we show another comparison of the noise as a function of the frequency in Fig. 2 below. On the left are data for a globar using aperture sizes of $22 \times 22$ microns, which is the practical limit with a conventional source, and on the right, data for the synchrotron with aperture sizes of $6 \times 6$ microns. The RMS (\%) is below $5 \times 10^{-3}$ between 4000 and $1300 \mathrm{~cm}^{-1}$ for both sources. The lost of signal below $1700 \mathrm{~cm}^{-1}$ is mainly due to the combination of diffraction effect and refractive index variation for this frequency range, the latter being the most important. However, an adequate retuning of the condenser could increase significantly the signal to noise ratio in this specific frequency range. If attention has to be paid in this region, less dispersive substrates like $\mathrm{KBr}$ would be better.
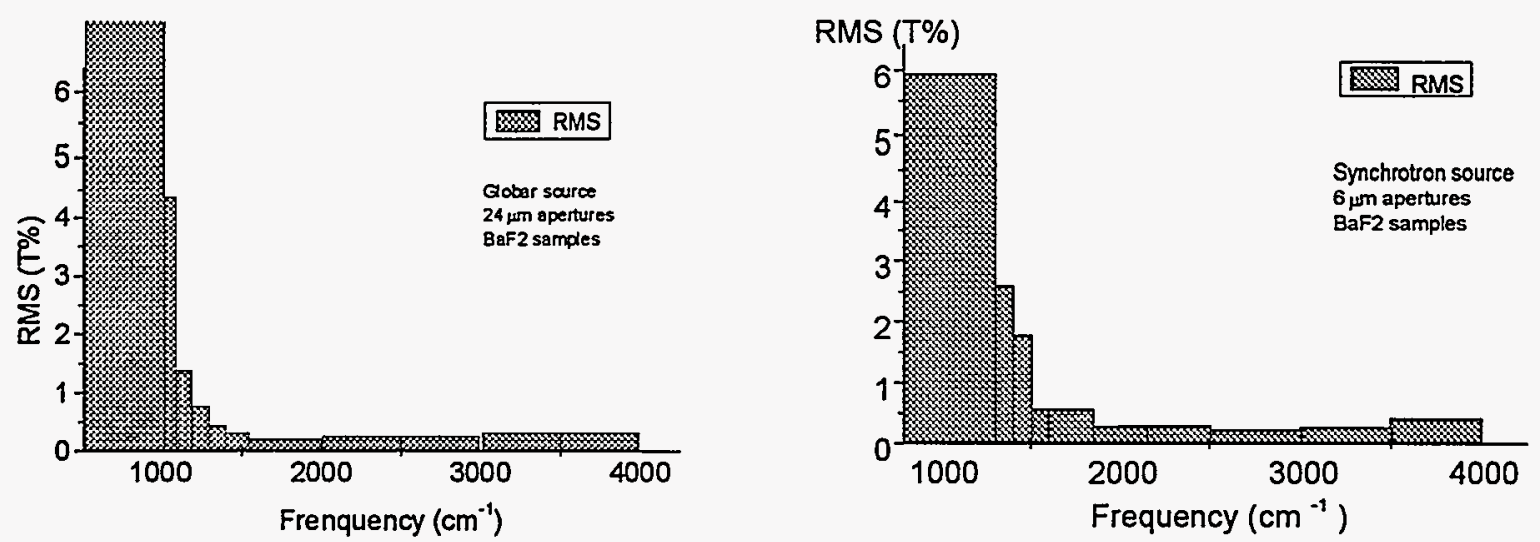

Figure 2 : Noise as a function of frequency for synchrotron and globar infrared sources (note the aperture sizes are different).

\section{CHEMICAL IMAGING OF A HAIR}

The ultimate goal of these experiments which map the chemical microstructure of hair is to study the interaction of chemical reagents. Initially, and as a control, a characterization of hair free from any chemical treatments is also needed and those results are presented here. This work is being done in collaboration with the Yves Rocher company.

From the morphological point of view, the human hair shaft structure consists of three main components (figure 4). The hair is typically composed of an inner core, the medulla ( $\sim 10 \mu \mathrm{m}$ diameter), which is surrounded by the cortex which constitutes the main part of the bulk of the fiber. The outer layer, which is exposed to the environment, is the cuticle with an average thickness of 5-10 $\mu \mathrm{m}$.

Chemically, hair mainly consists of keratin, a protein highly cross-linked by the amino acid cystine. The infrared frequencies characteristic of the intramolecular vibrations of this protein ${ }^{5}$ in the mid-IR $\left(4000-1000 \mathrm{~cm}^{-1}\right)$ are given in table 1 and agree well with those seen in a spectrum of hair.(figure 3 ). ${ }^{2.0}$

\begin{tabular}{|c|c|c|}
\hline & $v(\mathrm{~cm}-1)$ & comments \\
\hline$v \mathrm{NH}$ & 3290 & Amide A \\
\hline$v \mathrm{NH}$ & 3063 & Amide B \\
\hline$v \mathrm{CH}$ & $3000-2800$ & \\
\hline$v \mathrm{C}=\mathrm{O}$ & 1650 & Amide I \\
\hline$v \mathrm{CN}$ and $\delta \mathrm{NH}$ & 1540 & Amide 2 \\
& & \\
\hline
\end{tabular}

Table 1: vibration of the melanin protein ${ }^{5}$

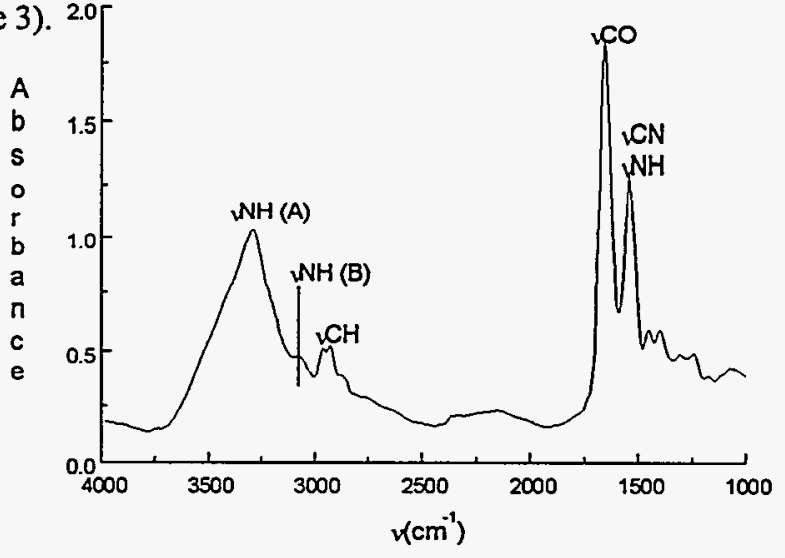

Figure 3 : IR spectrum of a hair 


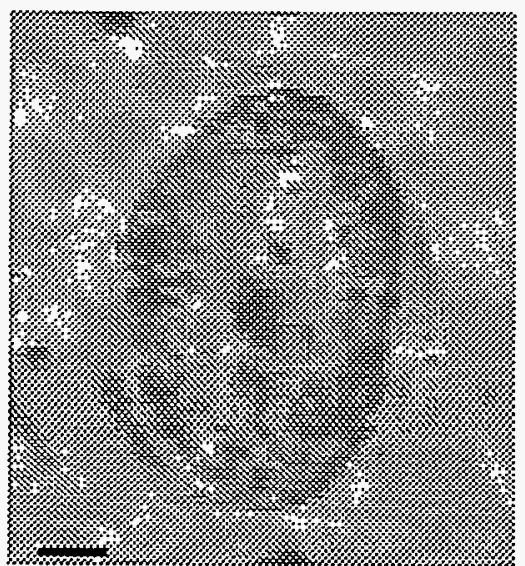

$15 \mu \mathrm{m}$
Samples were prepared by embedding hairs in polymer blocks and afterwards slicing with a microtome. The cross-sectioned cuts were on the order of $6 \mu \mathrm{m}$ thick (figure 4). The polymer was chosen to have vibrational features away from those of the hair sample.

Figure 4: cross-section of the hair (optical image)

The hair section shown in figure 3 was spectroscopically mapped with a lateral resolution of $6 \mu \mathrm{m}$. The integrated absorbance of the amide $\mathrm{A}$ band $\left(3290 \mathrm{~cm}^{-1}\right)$ was then extracted and a contour map showing the absorbance of this band as a function of position in the sample is shown in figure 5. Since this band is present throughout the hair, but at differing concentrations in the various components, the cuticle, cortex and medulla are all revealed in this map. The variations in absorbance which give the contrast are consistent with the differences in protein composition between these components ${ }^{5}$. The next step of this work will be to study the influence of dyes and cosmetic reagents on the structure of hair samples.

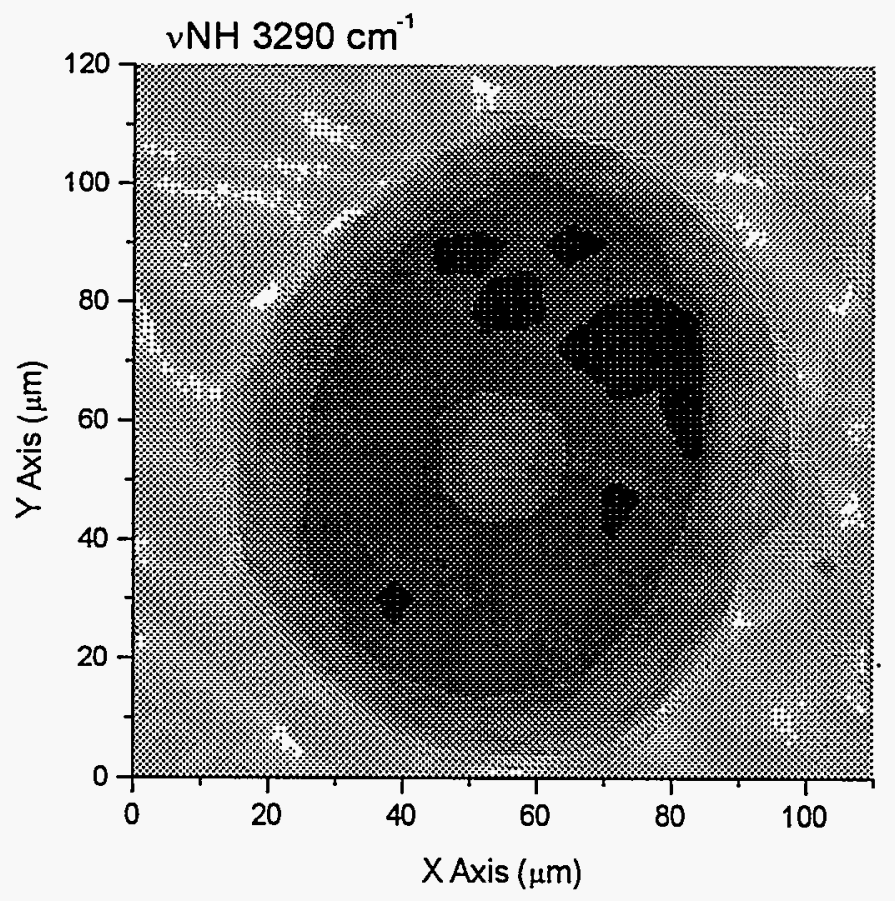

Figure 5 : Absorbance contour map of a hair for the amide $A$ band $\left(3290 \mathrm{~cm}^{-1}\right)$. Darker region represent lower absorbance.

\section{CHARACTERIZATION OF BITUMEN-POLYMER MIXTURES}

Bitumens are the heavy organic fraction of petroleum products. The main industrial application of this material is road surfaces. For this application, polymers are added to the bitumen to improve the mechanical properties. These in turn depend on the homogeneity of the mixture. Two ways of preparing the mixture can be used:

-One where the polymer is only mechanically mixed with the matrix of bitumen;

-a second one where the polymer chemically reacted with the bitumen.

The concentration of the polymer in the bitumen (5\%) is identical for both mixtures. 
We have characterized the relative distribution of the polymers in the bitumen matrix for the two different preparations.

For sampling, the bitumen-polymer mixture is first heated in a oven at $353 \mathrm{~K}$ and then spread on a $\mathrm{BaF}_{2}$ pellet of $2 \mathrm{~mm}$ thick. We obtain a film of which thickness is hardly under accurate control.

The mapping experiments were made in transmission mode with $2 \mathrm{~cm}^{-1}$ resolution and 128 accumulations per spectrum with a lateral resolution of 8 microns. Figure 6 shows the $\mathbb{R}$ spectrum of the mecanically mixed Bitumen-polymer. The $1377 \mathrm{~cm}^{-}$ ${ }^{\prime}$ band is a signature for the bitumen and the $967 \mathrm{~cm}^{-}$ ' band is assigned to the polymer. After mapping, the integrated absorbance (IA) of both bands has been calculated as a function of the position. As the thickness of the sample is not homogeneous, the ratios of these values (IA $967 \mathrm{~cm}^{-1} /$ IA $1377 \mathrm{~cm}^{-1}$ )

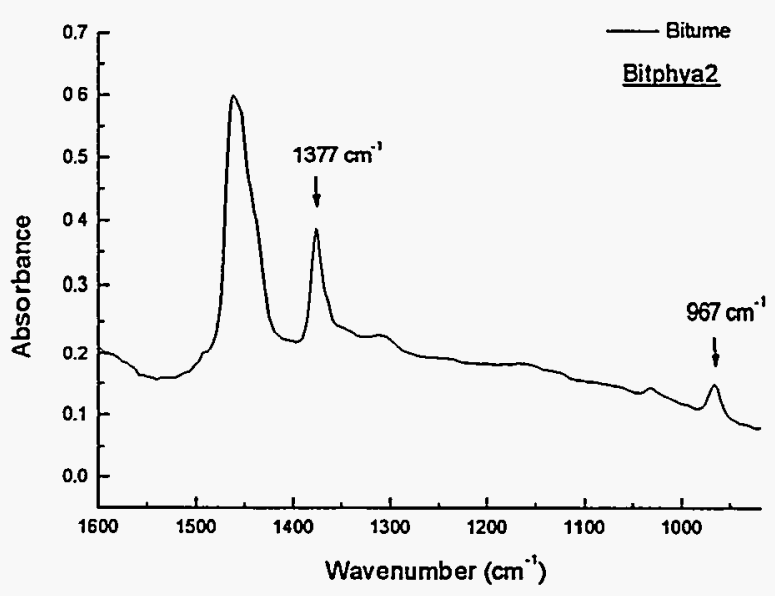
was calculated for each position.

Figure $6:$ IR spectrum of the mecanically mixed Bitumen-polymer

The image obtained is shown figure 7. The colors represent increments of $1.1 \%$ variation of relative concentration of polymer in the matrix of bitumen. The spatial resolution is limited by the diffraction limit of the wavelength of the polymer band at 10.3 microns. Therefore we are able to observe heterogeneity of the relative concentration of the polymer of $9 \%$ in the bitumen for the mechanically mixed sample. This scale of heterogeneity (order 10 microns) is impossible to observe by using a conventional source.

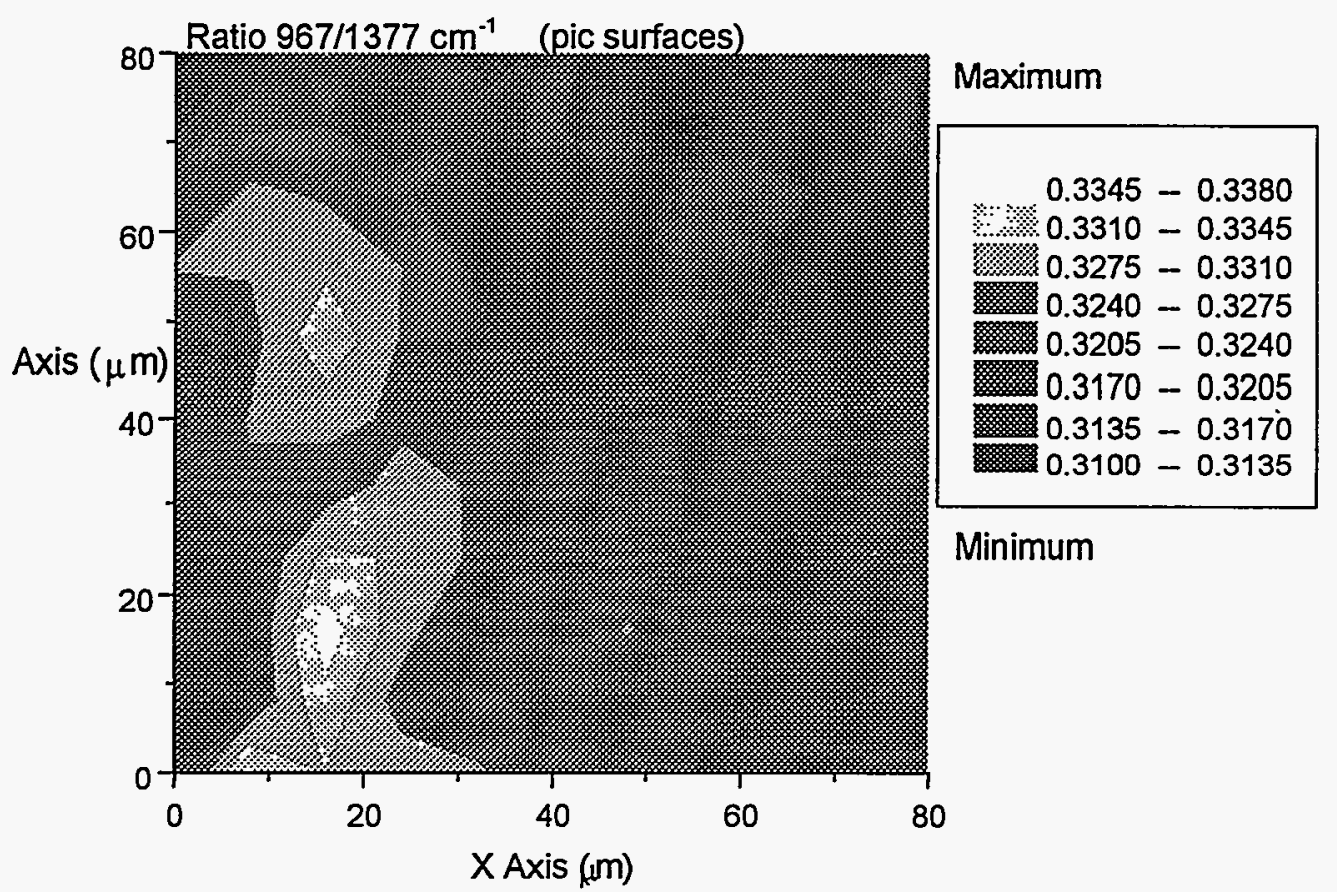

Figure 7: Absorbance contour map of a mecanically prepared bitume-polymer mixture for the ratio of the $967 \mathrm{~cm}^{-1}$ band (polymer) and the $1377 \mathrm{~cm}^{-1}$ band (bitumen). Darker region represent lower absorbance. 
We have also tried to make a similar map for the mixture made chemically, but the polymer band was weaker due to the chemical process. The intensity of the band is now too weak to allow a quantification of the relative proportion of both . phases (figure 8). Nevertheless a linear mapping of this mixture shows that this signature is still present everywhere even if it is weak (figure 9).

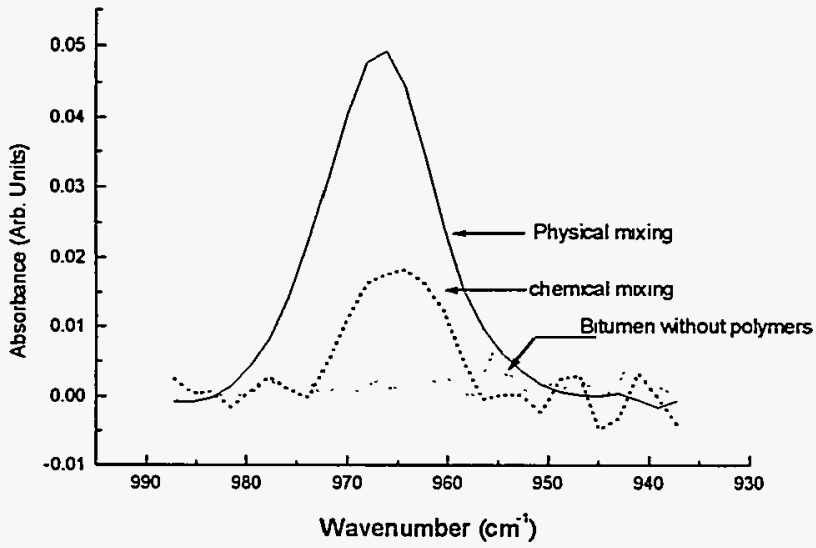

Figure $8: 967 \mathrm{~cm}^{-1}$ band of the polymer in the different mixtures mixture

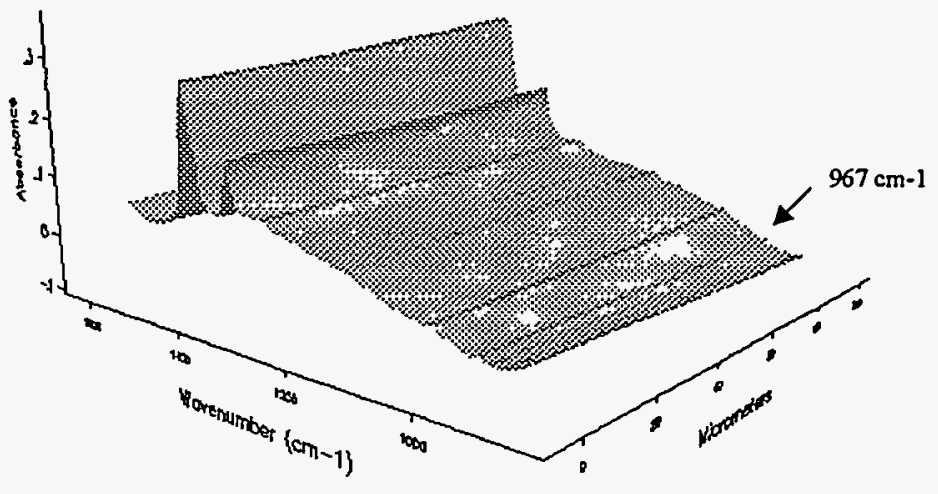

figure 9 : linear mapping of the chemical

\section{CHARACTERIZATION OF FLUORINATED POLYMER MULTILAYER}

Polymer multilayers are widely used in the chemical industry and building trade. A better knowledge of the polymer structure is essential if their mechanical strengths and longevities are to be improved. We have studied a multilayer comprising a PVDF polymer "sandwiched" with a PVC polymer and with a tie layer. Different industrial process (thermal and mechanical process) are used to prepare the multilayers. Those processes induce different behavior during ageing of the material. The characterization of the interfaces of the different multilayer has been made.

The sample is formed by two layer of PVDF polymer (thickness 25 and 12 microns) and a layer of paste (thickness 12 microns -Polymer P-) on a substrate of PVC. Due to the fact that the interface itself is of interest, the synchrotron is necessary to perform accurate chemical contrast images of this region.

Cross-sections of the multilayered polymers were prepared using a microtome, the samples being 10 microns thick. Linear mapping across the layers of the film was recorded at 3 microns intervals using $6 \times 6$ microns dual-confocal-apertures. Spectra were collected over a range of $3000-900 \mathrm{~cm}^{-1}$ with a resolution of $4 \mathrm{~cm}^{-1}$, and 128 scans were coadded for each spectrum. The chemical contrast image is shown on figure 10. Variations of composition across the layers are detected. More precisely, these results point out that the paste, characterized by the $1731 \mathrm{~cm}^{-1}$ band $(C=O$ vibration) diffuses into the PVDF polymer for at least 25 microns. Therefore, the barrier role of the interface of the two layers of PVDF polymer is shown. Due to the presence of a band of additives of the PVC at the same frequency, it is not possible to draw conclusive lines concerning the diffusion of the paste in the PVC. Due to the lack of contrast, this kind of information would have been impossible to get by optical microscopy.

The diffusion of the paste in the PVDF layer has been also observed when the multilayer is prepared by mechanical process. The microscope will now be used to study the influence of ageing on the structure of polymer samples. 


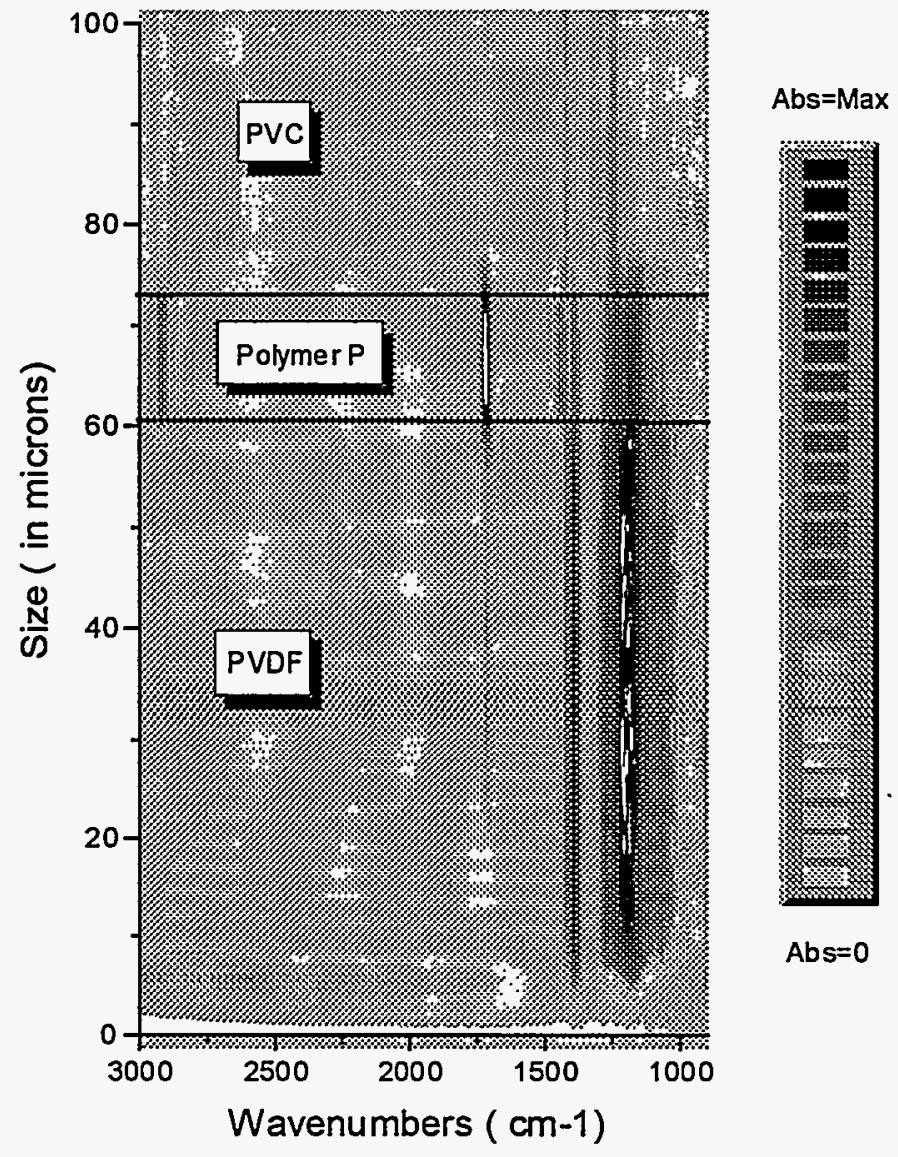

Figure 10 : Absorbance contour linear map of the fluorinated polymer multilayer obtained by mechanical process. Darker region represent higher absorbance.

\section{CONCLUSIONS}

By improving the spatial resolution of infrared microspectroscopy down to few microns, synchrotron radiation allows structural analysis at this level. Consequently, chemical identification of small samples and defects or composition gradients are possible. Chemical differences in the hair structure is shown, characterization of a polymer-bitumen mixture at several microns resolution is realized and heterogeneity of the mixture is pointed out. Furthermore, a more accurate description of polymer multilayer is demonstrated.

\section{ACKNOWLEDGEMENTS}

We are very grateful to Gwyn Williams for his assistance and very fruitful discussions and Jean-Marc Mouchon and Marc Piscaglia for the material preparation.

The results presented in this work are part of an ongoing study supported by Elf Aquitaine company.

The National Synchrotron Light Source is supported by the United States Department of Energy under contract DE-AC02$76 \mathrm{CH} 00016$. 


\section{REFERENCES}

1. J. E. Katon, G. E. Pacey and J. F. O'Keefe, "Vibrational molecular microspectroscopy", Am. Chem. Soc. 58, pp. 1-7. 1986.

2. R. G. Messerschmit and M. A. Harthcock, "Infrared microspectroscopy theory and applications 6, R. G. Messerschmit and M. A. Harthcock editors, New York and Basel, pp. 41-274. 1988.

3. G. L. Carr, A. Hanfland, and G. P. Williams, "Midinfrared beamline at the national Synchrotron Light Source port U2B", Rev. Sci. Instrum. 66 (2), pp. 1643-1645. 1995.

4. G. L. Carr, A. Hanfland, and G. P. Williams, "Performance of an infrared microspectrometer at the NSLS", Rev. Sci. Instrum. 66, pp. 1490-1492. 1995.

5. L. E. Jordana, K. P. Ghiggino, I. H. Leaver, C. G. Barraclough and P. Cole-Clarke, "Depth profile analysis of keratin fibers by FTIR photoacoustic spectroscopy", Appl. Spec. 48, pp. 44-49. 1994. 